\title{
Positive Solutions for Some Competitive Fractional Systems in Bounded Domains
}

\author{
Imed Bachar, ${ }^{1}$ Habib Mâagli, ${ }^{2}$ and Noureddine Zeddini ${ }^{2}$ \\ ${ }^{1}$ Mathematics Department, College of Science, King Saud University, P.O. Box 2455, Riyadh 11451, Saudi Arabia \\ ${ }^{2}$ Department of Mathematics, College of Sciences and Arts, King Abdulaziz University, Rabigh Campus, P.O. Box 344, \\ Rabigh 21911, Saudi Arabia
}

Correspondence should be addressed to Noureddine Zeddini; noureddine.zeddini@ipein.rnu.tn

Received 18 January 2013; Accepted 19 March 2013

Academic Editor: Nelson Merentes

Copyright (C) 2013 Imed Bachar et al. This is an open access article distributed under the Creative Commons Attribution License, which permits unrestricted use, distribution, and reproduction in any medium, provided the original work is properly cited.

Using some potential theory tools and the Schauder fixed point theorem, we prove the existence and precise global behavior of positive continuous solutions for the competitive fractional system $\left(-\Delta_{\mid D}\right)^{\alpha / 2} u+p(x) u^{\sigma} v^{r}=0,\left(-\Delta_{\mid D}\right)^{\alpha / 2} v+q(x) u^{s} v^{\beta}=0$ in a bounded $C^{1,1}$-domain $D$ in $\mathbb{R}^{n}(n \geq 3)$, subject to some Dirichlet conditions, where $0<\alpha<2, \sigma, \beta \geq 1, s, r \geq 0$. The potential functions $p, q$ are nonnegative and required to satisfy some adequate hypotheses related to the Kato class of functions $K_{\alpha}(D)$.

\section{Introduction and Statement of Main Results}

Let $D$ be a bounded $C^{1,1}$-domain in $\mathbb{R}^{n}(n \geq 3)$ and $\Delta_{\mid D}$ be the Dirichlet Laplacian in $D$. The fractional power $-\left(-\Delta_{\mid D}\right)^{\alpha / 2}$, $0<\alpha<2$, of the negative Dirichlet Laplacian is a very useful object in analysis and partial differential equations; see, for instance, $[1,2]$. There is a Markov process $Z_{\alpha}^{D}$ corresponding to $-\left(-\Delta_{\mid D}\right)^{\alpha / 2}$ which can be obtained as follows: we first kill the Brownian motion $X$ at $\tau_{D}$, the first exit time of $X$ from the domain $D$, and then we subordinate this killed Brownian motion using the $\alpha / 2$-stable subordinator $T_{t}$ starting at zero. For more description of the process $Z_{\alpha}^{D}$ and the development of its potential theory, we refer to [3-6].

In this paper, we will exploit these potential theory tools to study the existence of positive solutions for some nonlinear systems of fractional differential equations. More precisely, we fix two positive continuous functions $\varphi$ and $\psi$ on $\partial D$, and we will deal with the existence of positive continuous solutions (in the sense of distributions) for the following competitive fractional system:

$$
\begin{array}{ll}
\left(-\Delta_{\mid D}\right)^{\alpha / 2} u+p(x) u^{\sigma} v^{r}=0 & \text { in } D, \\
\left(-\Delta_{\mid D}\right)^{\alpha / 2} v+q(x) u^{s} v^{\beta}=0 & \text { in } D,
\end{array}
$$

$$
\begin{aligned}
& \lim _{x \rightarrow z \in \partial D} \frac{u(x)}{M_{\alpha}^{D} 1(x)}=\varphi(z), \\
& \lim _{x \rightarrow z \in \partial D} \frac{v(x)}{M_{\alpha}^{D} 1(x)}=\psi(z),
\end{aligned}
$$

where $\sigma, \beta \geq 1, s, r \geq 0,0<\alpha<2$ and the nonnegative potential functions $p, q$ are required to satisfy some adequate hypotheses related to the Kato class of functions $K_{\alpha}(D)$ (see Definition 1). The function $M_{\alpha}^{D} 1(x)$ is defined by

$$
M_{\alpha}^{D} 1(x)=\frac{1-\alpha / 2}{\Gamma(\alpha / 2)} \int_{0}^{\infty} t^{-2+(\alpha / 2)}\left(1-P_{t}^{D} 1(x)\right) d t,
$$

where $\left(P_{t}^{D}\right)_{t>0}$ is the semigroup corresponding to the killed Brownian motion upon exiting $D$.

We recall that in $[6$, Remark 3.3], the authors have proved the existence of a constant $C>0$ such that for each $x \in D$,

$$
\frac{1}{C}(\delta(x))^{\alpha-2} \leq M_{\alpha}^{D} 1(x) \leq C(\delta(x))^{\alpha-2},
$$

where $\delta(x)$ denotes the Euclidian distance from $x$ to the boundary of $D$.

In the classical case (i.e., $\alpha=2$ ), there is a large amount of literature dealing with the existence, nonexistence, and qualitative analysis of positive solutions for the problems related 
to (1); see for example, the papers of Cîrstea and Rădulescu [7], Ghanmi et al. [8], Ghergu and Rădulescu [9], Lair and Wood [10, 11], Mu et al. [12], and references therein. In these works, various existence results of positive bounded solutions or positive blowing up ones (called also large solutions) have been established, and a precise global behavior is given. We note also that several methods have been used to treat these systems such as sub- and supersolutions method, variational method, and topological methods. In [11], the authors studied the system (1) with $\alpha=2$ in the case $\sigma=\beta=0, s>0, r>$ 0 , and $p, q$ are nonnegative continuous and not necessarily radial. They showed that entire positive bounded solutions exist if $p$ and $q$ satisfy the following condition:

$$
p(x)+q(x) \leq C|x|^{-(2+\delta)},
$$

for some positive constant $\delta$ and $|x|$ large.

These results have been extended recently by Alsaedi et al. in [13], in the case $\alpha=2, \sigma, \beta \geq 1, s>0, r>0$, where the authors established the existence of a positive continuous bounded solution for (1).

In this paper, first, we aim at proving the existence and uniqueness of a positive continuous solution (in the sense of distributions) for the following scalar equation:

$$
\begin{gathered}
\left(-\Delta_{\mid D}\right)^{\alpha / 2} u+p_{0}(x) u^{\gamma}=0 \quad \text { in } D, \\
u>0 \quad \text { in } D, \\
\lim _{x \rightarrow z \in \partial D} \frac{u(x)}{M_{\alpha}^{D} 1(x)}=\varphi(z),
\end{gathered}
$$

where $\gamma \geq 1$ and $p_{0}$ is a nonnegative Borel measurable function in $D$ satisfying the following.

$\left(\mathbf{H}_{1}\right)$ The function $x \rightarrow(\delta(x))^{(\alpha-2)(\gamma-1)} p_{0}(x) \in K_{\alpha}(D)$.

The class of functions $K_{\alpha}(D)$, is defined by means of the Green function $G_{\alpha}^{D}$ of $Z_{\alpha}^{D}$ as follows.

Definition 1 (see [14]). A Borel measurable function $q$ in $D$ belongs to the Kato class of functions $K_{\alpha}(D)$ if

$$
\lim _{r \rightarrow 0}\left(\sup _{x \in D} \int_{(|x-y| \leq r) \cap D} \frac{\delta(y)}{\delta(x)} G_{\alpha}^{D}(x, y)|q(y)| d y\right)=0 .
$$

It has been shown in [14], that

$$
x \longrightarrow(\delta(x))^{-\lambda} \in K_{\alpha}(D), \quad \text { for } \lambda<\alpha .
$$

For more examples of functions belonging to $K_{\alpha}(D)$, we refer to [14]. Note that for the classical case (i.e., $\alpha=2$ ), the class of functions $K_{2}(D)$ was introduced and studied in [15].

In order to state our existence result, we denote by $M_{\alpha}^{D} \varphi$ (see [3]) the unique positive continuous solution of

$$
\left(-\Delta_{\mid D}\right)^{\alpha / 2} u=0 \text { in } D \text { (in the sense of distributions). }
$$

$$
\lim _{x \rightarrow z \in \partial D} \frac{u(x)}{M_{\alpha}^{D} 1(x)}=\varphi(z) .
$$

Using some potential theory tools and an approximating sequence, we establish the following.
Theorem 2. Under hypothesis $\left(\mathbf{H}_{1}\right)$, the problem (5) has a unique positive continuous solution satisfying for each $x \in D$,

$$
c_{0} M_{\alpha}^{D} \varphi(x) \leq u(x) \leq M_{\alpha}^{D} \varphi(x),
$$

where the constant $c_{0} \in(0,1]$.

Using (7), hypothesis $\left(\mathbf{H}_{1}\right)$ is satisfied if $p_{0}$ verifies the following condition: there exists a constant $C>0$, such that for each $x \in D$,

$$
p_{0}(x) \leq \frac{C}{(\delta(x))^{\tau}}, \quad \text { with } \tau+(2-\alpha)(\gamma-1)<\alpha .
$$

Next, we exploit the result of Theorem 2, to prove the existence of a positive continuous solution $(u, v)$ to the system (1). To this end, we assume the following hypothesis.

$\left(\mathbf{H}_{2}\right)$ The functions $p, q$ are two nonnegative Borel measurable functions such that

$$
\begin{aligned}
& x \longrightarrow(\delta(x))^{(\alpha-2)(\sigma+r-1)} p(x) \in K_{\alpha}(D), \\
& x \longrightarrow(\delta(x))^{(\alpha-2)(\beta+s-1)} q(x) \in K_{\alpha}(D) .
\end{aligned}
$$

Then, by using Schauder's fixed point theorem, we prove the following.

Theorem 3. Under assumption $\left(\mathbf{H}_{2}\right)$, the problem (1) has a positive continuous solution $(u, v)$ satisfying for each $x \in D$,

$$
\begin{aligned}
& c_{1} M_{\alpha}^{D} \varphi(x) \leq u(x) \leq M_{\alpha}^{D} \varphi(x), \\
& c_{2} M_{\alpha}^{D} \psi(x) \leq v(x) \leq M_{\alpha}^{D} \psi(x),
\end{aligned}
$$

where $c_{1} \in(0,1]$ and $c_{2} \in(0,1]$.

We note that contrary to the classical case $\alpha=2$, in our situation, the solution blows up on the boundary of $D$.

The content of this paper is organized as follows. In Section 2, we collect some properties of functions belonging to the Kato class of functions $K_{\alpha}(D)$, which are useful to establish our results. Our main results are proved in Section 3.

As usual, let $B^{+}(D)$ be the set of nonnegative Borel measurable functions in $D$. We denote by $C_{0}(D)$ the set of continuous functions in $\bar{D}$ vanishing continuously on $\partial D$. Note that $C_{0}(D)$ is a Banach space with respect to the uniform norm $\|u\|_{\infty}=\sup _{x \in D}|u(x)|$. When two positive functions $f$ and $g$ are defined on a set $S$, we write $f \approx g$ if the twosided inequality $(1 / C) g \leq f \leq C g$ holds on $S$. We define the potential kernel $G_{\alpha}^{D}$ of $Z_{\alpha}^{D}$ by

$$
G_{\alpha}^{D} f(x):=\int_{D} G_{\alpha}^{D}(x, y) f(y) d y, \quad \text { for } f \in B^{+}(D), x \in D .
$$

Finally, let us recall some potential theory tools that are needed, and we refer to $[14,16,17]$ for more details. For $q \epsilon$ $B^{+}(D)$, we define the kernel $V_{q}$ on $B^{+}(D)$ by

$$
V_{q} f(x):=\int_{0}^{\infty} \widetilde{E}^{x}\left(e^{-\int_{0}^{t} q\left(Z_{\alpha}^{D}(s)\right) d s} f\left(Z_{\alpha}^{D}(t)\right)\right) d t, \quad x \in D,
$$


with $V_{0}:=V=G_{\alpha}^{D}$, where $\widetilde{E}^{x}$ stands for the expectation with respect to $Z_{\alpha}^{D}$ starting from $x$. If $q$ satisfies $V q<\infty$, we have the following resolvent equation:

$$
V=V_{q}+V_{q}(q V)=V_{q}+V\left(q V_{q}\right) .
$$

In particular, if $u \in B^{+}(D)$ is such that $V(q u)<\infty$, then we have

$$
\left(I-V_{q}(q .)\right)(I+V(q .)) u=(I+V(q .))\left(I-V_{q}(q .)\right) u=u .
$$

\section{The Kato Class of Functions $K_{\alpha}(D)$}

Proposition 4 (see [14]). Let $q$ be a function in $K_{\alpha}(D)$, then we have the following.

(i) $a_{\alpha}(q):=\sup _{x, y \in D} \int_{D}\left(G_{\alpha}^{D}(x, z) G_{\alpha}^{D}(z, y) / G_{\alpha}^{D}(x, y)\right)$ $|q(z)| d z<\infty$.

(ii) Let $h$ be a positive excessive function on $D$ with respect to $Z_{\alpha}^{D}$. Then, we have

$$
\int_{D} G_{\alpha}^{D}(x, y) h(y)|q(y)| d y \leq a_{\alpha}(q) h(x) .
$$

Furthermore, for each $x_{0} \in \bar{D}$, we have

$$
\lim _{r \rightarrow 0}\left(\sup _{x \in D} \frac{1}{h(x)} \int_{B\left(x_{0}, r\right) \cap D} G_{\alpha}^{D}(x, y) h(y)|q(y)| d y\right)=0 .
$$

(iii) The function $x \rightarrow(\delta(x))^{\alpha-1} q(x)$ is in $L^{1}(D)$.

The next two lemmas will play a special role.

Lemma 5. Let $q$ be a nonnegative function in $K_{\alpha}(D)$ and $h$ be a positive finite excessive function on $D$ with respect to $Z_{\alpha}^{D}$. Then, for all $x \in D$, we have

$$
\exp \left(-a_{\alpha}(q)\right) h(x) \leq h(x)-V_{q}(q h)(x) \leq h(x) .
$$

Proof. Let $h$ be a positive finite excessive function on $D$ with respect to $Z_{\alpha}^{D}$. Then, by [18, Chapter II, proposition 3.11], there exists a sequence $\left(f_{k}\right)_{k}$ of nonnegative measurable functions in $D$ such that $h=\sup _{k} V f_{k}$. Let $x \in D$ and $k \in \mathbb{N}$ such that $0<V f_{k}<\infty$. Consider $\theta(t)=V_{t q} f_{k}(x)$, for $t \geq 0$. Then, by (14), the function $\theta$ is completely monotone on $[0, \infty)$, and so from the Hölder inequality and $[19$, Theorem $12 \mathrm{a}]$, the function $\log \theta$ is convex on $[0, \infty)$. This implies that

$$
\theta(0) \leq \theta(1) \exp \left(-\frac{\theta^{\prime}(0)}{\theta(0)}\right)
$$

That is

$$
V f_{k}(x) \leq V_{q} f_{k}(x) \exp \left(\frac{V\left(q V f_{k}\right)(x)}{V f_{k}(x)}\right) .
$$

Hence, it follows from (17) that

$$
V f_{k}(x) \leq V_{q} f_{k}(x) \exp \left(a_{\alpha}(q)\right)
$$

Consequently, from (15) we obtain

$$
\exp \left(-a_{\alpha}(q)\right) V f_{k}(x) \leq V f_{k}(x)-V_{q}\left(q V f_{k}\right)(x) \leq V f_{k}(x) .
$$

The result holds by letting $k \rightarrow \infty$.

Lemma 6. Let $q$ be a nonnegative function in $K_{\alpha}(D)$, then the family of functions

$$
\Lambda_{q}=\left\{\frac{1}{M_{\alpha}^{D} \varphi(x)} \int_{D} G_{\alpha}^{D}(x, y) M_{\alpha}^{D} \varphi(y) f(y) d y,|f| \leq q\right\}
$$

is uniformly bounded and equicontinuous in $\bar{D}$. Consequently, $\Lambda_{q}$ is relatively compact in $C_{0}(D)$.

Proof. Taking $h \equiv M_{\alpha}^{D} \varphi$ in (17), we deduce that for $|f| \leq q$ and $x \in D$, we have

$$
\begin{aligned}
& \left|\int_{D} \frac{G_{\alpha}^{D}(x, y)}{M_{\alpha}^{D} \varphi(x)} M_{\alpha}^{D} \varphi(y) f(y) d y\right| \\
& \quad \leq \int_{D} \frac{G_{\alpha}^{D}(x, y)}{M_{\alpha}^{D} \varphi(x)} M_{\alpha}^{D} \varphi(y) q(y) d y \leq a_{\alpha}(q)<\infty .
\end{aligned}
$$

So, the family $\Lambda_{q}$ is uniformly bounded.

Next, we aim at proving that the family $\Lambda_{q}$ is equicontinuous in $\bar{D}$.

First, we recall the following interesting sharp estimates on $G_{\alpha}^{D}$, which is proved in [5]:

$$
G_{\alpha}^{D}(x, y) \approx|x-y|^{\alpha-n} \min \left(1, \frac{\delta(x) \delta(y)}{|x-y|^{2}}\right) .
$$

Let $x_{0} \in \bar{D}$ and $\varepsilon>0$. By (18), there exists $r>0$ such that

$$
\sup _{z \in D} \frac{1}{M_{\alpha}^{D} \varphi(z)} \int_{B\left(x_{0}, 2 r\right) \cap D} G_{\alpha}^{D}(z, y) M_{\alpha}^{D} \varphi(y) q(y) d y \leq \frac{\varepsilon}{2} .
$$


If $x_{0} \in D$ and $x, x^{\prime} \in B\left(x_{0}, r\right) \cap D$, then for $|f| \leq q$, we have

$$
\begin{aligned}
& \mid \int_{D} \frac{G_{\alpha}^{D}(x, y)}{M_{\alpha}^{D} \varphi(x)} M_{\alpha}^{D} \varphi(y) f(y) d y \\
& \quad-\int_{D} \frac{G_{\alpha}^{D}\left(x^{\prime}, y\right)}{M_{\alpha}^{D} \varphi\left(x^{\prime}\right)} M_{\alpha}^{D} \varphi(y) f(y) d y \mid \\
& \leq \int_{D}\left|\frac{G_{\alpha}^{D}(x, y)}{M_{\alpha}^{D} \varphi(x)}-\frac{G_{\alpha}^{D}\left(x^{\prime}, y\right)}{M_{\alpha}^{D} \varphi\left(x^{\prime}\right)}\right| M_{\alpha}^{D} \varphi(y) q(y) d y \\
& \leq 2 \sup _{z \in D} \int_{B\left(x_{0}, 2 r\right) \cap D} \frac{1}{M_{\alpha}^{D} \varphi(z)} G_{\alpha}^{D}(z, y) M_{\alpha}^{D} \varphi(y) q(y) d y \\
& \quad+\int_{\left(\left|x_{0}-y\right| \geq 2 r\right) \cap D}\left|\frac{G_{\alpha}^{D}(x, y)}{M_{\alpha}^{D} \varphi(x)}-\frac{G_{\alpha}^{D}\left(x^{\prime}, y\right)}{M_{\alpha}^{D} \varphi\left(x^{\prime}\right)}\right| M_{\alpha}^{D} \varphi(y) q(y) d y \\
& \leq \varepsilon+\int_{\left(\left|x_{0}-y\right| \geq 2 r\right) \cap D}\left|\frac{G_{\alpha}^{D}(x, y)}{M_{\alpha}^{D} \varphi(x)}-\frac{G_{\alpha}^{D}\left(x^{\prime}, y\right)}{M_{\alpha}^{D} \varphi\left(x^{\prime}\right)}\right| M_{\alpha}^{D} \varphi(y) q(y) d y .
\end{aligned}
$$

On the other hand, for every $y \in B^{c}\left(x_{0}, 2 r\right) \cap D$ and $x, x^{\prime} \in$ $B\left(x_{0}, r\right) \cap D$, by using (26) and the fact that $M_{\alpha}^{D} \varphi(z) \approx$ $(\delta(z))^{\alpha-2}$, we have

$$
\begin{aligned}
& \left|\frac{1}{M_{\alpha}^{D} \varphi(x)} G_{\alpha}^{D}(x, y)-\frac{1}{M_{\alpha}^{D} \varphi\left(x^{\prime}\right)} G_{\alpha}^{D}\left(x^{\prime}, y\right)\right| M_{\alpha}^{D} \varphi(y) \\
& \quad \leq C(\delta(y))^{\alpha-1} .
\end{aligned}
$$

Now since $x \rightarrow\left(1 / M_{\alpha}^{D} \varphi(x)\right) G_{\alpha}^{D}(x, y)$ is continuous outside the diagonal and $q \in K_{\alpha}(D)$, we deduce by the dominated convergence theorem and Proposition 4 (iii) that

$$
\begin{gathered}
\int_{\left(\left|x_{0}-y\right| \geq 2 r\right) \cap D}\left|\frac{G_{\alpha}^{D}(x, y)}{M_{\alpha}^{D} \varphi(x)}-\frac{G_{\alpha}^{D}\left(x^{\prime}, y\right)}{M_{\alpha}^{D} \varphi\left(x^{\prime}\right)}\right| M_{\alpha}^{D} \varphi(y) q(y) d y \\
\longrightarrow 0 \quad \text { as }\left|x-x^{\prime}\right| \longrightarrow 0 .
\end{gathered}
$$

If $x_{0} \in \partial D$ and $x \in B\left(x_{0}, r\right) \cap D$, then we have

$$
\begin{aligned}
& \left|\int_{D} \frac{G_{\alpha}^{D}(x, y)}{M_{\alpha}^{D} \varphi(x)} M_{\alpha}^{D} \varphi(y) f(y) d y\right| \\
& \quad \leq \frac{\varepsilon}{2}+\int_{\left(\left|x_{0}-y\right| \geq 2 r\right) \cap D} \frac{G_{\alpha}^{D}(x, y)}{M_{\alpha}^{D} \varphi(x)} M_{\alpha}^{D} \varphi(y) q(y) d y .
\end{aligned}
$$

Now, since $G_{\alpha}^{D}(x, y) / M_{\alpha}^{D} \varphi(x) \rightarrow 0$ as $\left|x-x_{0}\right| \rightarrow 0$, for $\left|x_{0}-y\right| \geq 2 r$, then by the same argument as above, we get

$$
\begin{gathered}
\int_{\left(\left|x_{0}-y\right| \geq 2 r\right) \cap D} \frac{G_{\alpha}^{D}(x, y)}{M_{\alpha}^{D} \varphi(x)} M_{\alpha}^{D} \varphi(y) q(y) d y \longrightarrow 0 \\
\text { as }\left|x-x_{0}\right| \longrightarrow 0 .
\end{gathered}
$$

Consequently, by Ascoli's theorem, we deduce that $\Lambda_{q}$ is relatively compact in $C_{0}(D)$.

\section{Proofs of Theorems 2 and 3}

The next Lemma will be used for uniqueness.

Lemma 7 (see [14, Proposition 6]). Let $h \in B^{+}(D)$ and $v$ be a nonnegative excessive function on $D$ with respect to $Z_{\alpha}^{D}$. Let $z$ be a Borel measurable function in D such that $V(h|z|)<\infty$ and $v=z+V(h z)$. Then, $z$ satisfies

$$
0 \leq z \leq v
$$

Proof of Theorem 2. Let $\varphi$ be a positive continuous function on $\partial D$. We recall that on $D$, we have

$$
M_{\alpha}^{D} \varphi(x) \approx M_{\alpha}^{D} 1(x) \approx(\delta(x))^{\alpha-2} .
$$

Let $\widetilde{p_{0}}=\gamma\left(M_{\alpha}^{D} \varphi\right)^{\gamma-1} p_{0}$ and put $c_{0}=e^{-a_{\alpha}\left(\widetilde{p_{0}}\right)}$, where $a_{\alpha}\left(\widetilde{p_{0}}\right)$ is given by Proposition $4(\mathrm{i})$. Since by $\left(\mathbf{H}_{1}\right), \widetilde{p_{0}} \in K_{\alpha}(D)$, it follows from Proposition 4 that $V\left(\widetilde{p_{0}}\right) \leq a_{\alpha}\left(\widetilde{p_{0}}\right)<\infty$. Define the nonempty closed bounded convex $\Lambda$ by

$$
\Lambda=\left\{\omega \in B^{+}(D): c_{0} \leq \omega \leq 1\right\} .
$$

Let $T$ be the operator defined on $\Lambda$ by

$$
\begin{aligned}
T \omega:= & 1-\frac{1}{M_{\alpha}^{D} \varphi} V_{\widetilde{p_{0}}}\left(\widetilde{p_{0}} M_{\alpha}^{D} \varphi\right) \\
& +\frac{1}{M_{\alpha}^{D} \varphi} V_{\widetilde{p_{0}}}\left(\widetilde{p_{0}} \omega M_{\alpha}^{D} \varphi-p_{0}\left(\omega M_{\alpha}^{D} \varphi\right)^{\gamma}\right) .
\end{aligned}
$$

We claim that $T$ maps $\Lambda$ to itself. Indeed, for each $\omega \in \Lambda$, we have

$$
T \omega \leq 1-\frac{1}{M_{\alpha}^{D} \varphi} V_{\widetilde{p_{0}}}\left(p_{0}\left(\omega M_{\alpha}^{D} \varphi\right)^{\gamma}\right) \leq 1 .
$$

On the other hand, since the function $\widetilde{p_{0}} \omega M_{\alpha}^{D} \varphi-p_{0}$ $\left(\omega M_{\alpha}^{D} \varphi\right)^{\gamma} \geq 0$, we deduce by Lemma 5 with $h=M_{\alpha}^{D} \varphi$, that $T \omega \geq 1-\left(1 / M_{\alpha}^{D} \varphi\right) V_{\widetilde{p_{0}}}\left(\widetilde{p_{0}} M_{\alpha}^{D} \varphi\right) \geq c_{0}$. Hence, $T \Lambda \subset \Lambda$. Next, we aim at proving that $T$ is nondecreasing on $\Lambda$. To this end, we let $\omega_{1}, \omega_{2} \in \Lambda$ such that $\omega_{1} \leq \omega_{2}$. Using the fact that the function $t \rightarrow \gamma t-t^{\gamma}$ is nondecreasing on $[0,1]$, we deduce that

$$
\begin{aligned}
T \omega_{2} & -T \omega_{1} \\
= & \frac{1}{M_{\alpha}^{D} \varphi} V_{\widetilde{p_{0}}}\left(\widetilde{p_{0}} \omega_{2} M_{\alpha}^{D} \varphi-p_{0}\left(\omega_{2} M_{\alpha}^{D} \varphi\right)^{\gamma}\right) \\
& -\frac{1}{M_{\alpha}^{D} \varphi} V_{\widetilde{p_{0}}}\left(\widetilde{p_{0}} \omega_{1} M_{\alpha}^{D} \varphi-p_{0}\left(\omega_{1} M_{\alpha}^{D} \varphi\right)^{\gamma}\right) \\
= & \frac{1}{M_{\alpha}^{D} \varphi} V_{\widetilde{p_{0}}}\left(p_{0}\left(M_{\alpha}^{D} \varphi\right)^{\gamma}\left[\left(\gamma \omega_{2}-\omega_{2}^{\gamma}\right)-\left(\gamma \omega_{1}-\omega_{1}^{\gamma}\right)\right]\right)
\end{aligned}
$$$$
\geq 0 \text {. }
$$

Next, we define the sequence $\left(\omega_{k}\right)_{k \geq 0}$ by

$$
\begin{gathered}
\omega_{0}=1-\frac{1}{M_{\alpha}^{D} \varphi} V_{\widetilde{p_{0}}}\left(\widetilde{p_{0}} M_{\alpha}^{D} \varphi\right), \\
\omega_{k+1}=T \omega_{k} .
\end{gathered}
$$


Clearly $\omega_{0} \in \Lambda$ and $\omega_{1}=T \omega_{0} \geq \omega_{0}$. Thus, from the monotonicity of $T$, we deduce that

$$
c_{0} \leq \omega_{0} \leq \omega_{1} \leq \cdots \leq \omega_{k} \leq 1 .
$$

So, the sequence $\left(\omega_{k}\right)_{k \geq 0}$ converges to a measurable function $\omega \in \Lambda$. Therefore, by applying the monotone convergence theorem, we obtain

$$
\begin{aligned}
\omega= & 1-\frac{1}{M_{\alpha}^{D} \varphi} V_{\widetilde{p_{0}}}\left(\widetilde{p_{0}} M_{\alpha}^{D} \varphi\right) \\
& +\frac{1}{M_{\alpha}^{D} \varphi} V_{\widetilde{p_{0}}}\left(\widetilde{p_{0}} \omega M_{\alpha}^{D} \varphi-p_{0}\left(\omega M_{\alpha}^{D} \varphi\right)^{\gamma}\right) .
\end{aligned}
$$

Put $u=\omega M_{\alpha}^{D} \varphi$. Then, we have

$$
u=M_{\alpha}^{D} \varphi-V_{\widetilde{p_{0}}}\left(\widetilde{p_{0}} M_{\alpha}^{D} \varphi\right)+V_{\widetilde{p_{0}}}\left(\widetilde{p_{0}} u-p_{0} u^{\gamma}\right)
$$

or equivalently

$$
u-V_{\widetilde{p_{0}}}\left(\widetilde{p_{0}} u\right)=M_{\alpha}^{D} \varphi-V_{\widetilde{p_{0}}}\left(\widetilde{p_{0}} M_{\alpha}^{D} \varphi\right)-V_{\widetilde{p_{0}}}\left(p_{0} u^{\gamma}\right) .
$$

Observe that by Proposition 4(ii), we have $V\left(\widetilde{p_{0}} u\right)<\infty$. So, applying the operator $\left(I+V\left(\widetilde{p_{0}}.\right)\right)$ on both sides of $(43)$, we deduce by using (15) and (16) that

$$
u=M_{\alpha}^{D} \varphi-V\left(p_{0} u^{\gamma}\right) .
$$

Now, using $\left(\mathbf{H}_{1}\right)$ and similar argument as in the proof of Lemma 6, we prove that $x \rightarrow\left(1 / M_{\alpha}^{D} \varphi\right) V\left(p_{0} u^{\gamma}\right) \in C_{0}(D)$. So, $u$ is a continuous function in $D$, and $u$ is a solution of (5). It remains to prove the uniqueness of such a solution. Let $u$ be a continuous solution of (5). Since the function $x \rightarrow u(x) / M_{\alpha}^{D} 1(x)$ is continuous and positive in $D$ such that $\lim _{x \rightarrow z \in \partial D}\left(u(x) / M_{\alpha}^{D} 1(x)\right)=\varphi(z)$, it follows that $u(x) \approx$ $M_{\alpha}^{D} 1(x) \approx M_{\alpha}^{D} \varphi(x)$. Then, by using this fact and Lemma 6, we have

$$
\begin{gathered}
\left(-\Delta_{\mid D}\right)^{\alpha / 2}\left(u+V\left(p_{0} u^{\gamma}\right)\right)=0 \quad \text { in } D, \\
\lim _{x \rightarrow z \in \partial D} \frac{\left(u+V\left(p_{0} u^{\gamma}\right)\right)(x)}{M_{\alpha}^{D} 1(x)}=\varphi(z) \quad \text { in } D .
\end{gathered}
$$

So, from the uniqueness of the problem (8) (see [3]), we deduce that

$$
u+V\left(p_{0} u^{\gamma}\right)=M_{\alpha}^{D} \varphi \text { in } D .
$$

It follows that if $u$ and $v$ are two continuous solution of (5), then $z=v-u$ satisfies

$$
z+V\left(p_{0} h z\right)=0 \quad \text { in } D,
$$

where $h$ is the nonnegative measurable function defined in $D$ by

$$
h(x)= \begin{cases}\frac{v^{\gamma}-u^{\gamma}}{v-u}, & \text { if } u(x) \neq v(x), \\ 0, & \text { if } u(x)=v(x) .\end{cases}
$$

Since $V\left(p_{0} h|z|\right)<\infty$, we deduce by Lemma 7 that $z=0$, and so $u=v$.
Proof of Theorem 3. Let $\tilde{p}=\sigma\left(M_{\alpha}^{D} \varphi\right)^{\sigma-1}\left(M_{\alpha}^{D} \psi\right)^{r} p$ and $\widetilde{q}=$ $\beta\left(M_{\alpha}^{D} \psi\right)^{\beta-1}\left(M_{\alpha}^{D} \varphi\right)^{s} q$.

Put $c_{1}=e^{-a_{\alpha}(\tilde{p})}, c_{2}=e^{-a_{\alpha}(\tilde{q})}$. Note that from $\left(\mathbf{H}_{2}\right)$ and Proposition 4 , we have $a_{\alpha}(\tilde{p})<\infty$ and $a_{\alpha}(\widetilde{q})<\infty$. Consider the nonempty closed convex set $\Gamma$ defined by

$$
\Gamma=\left\{(y, z) \in C(\bar{D}) \times C(\bar{D}): c_{1} \leq y \leq 1, c_{2} \leq z \leq 1\right\} .
$$

Let $T$ be the operator defined on $\Gamma$ by $T(y, z):=(\omega, \theta)$, such that $\left(\widetilde{u}=\omega M_{\alpha}^{D} \varphi, \widetilde{v}=\theta M_{\alpha}^{D} \psi\right)$ is the unique positive continuous solution of the following problem:

$$
\begin{gathered}
\left(-\Delta_{\mid D}\right)^{\alpha / 2} \widetilde{u}+\left(\left(M_{\alpha}^{D} \psi\right)^{r} z^{r} p\right)(x) \widetilde{u}^{\sigma}=0 \text { in } D, \\
\left(-\Delta_{\mid D}\right)^{\alpha / 2} \widetilde{v}+\left(\left(M_{\alpha}^{D} \varphi\right)^{s} y^{s} q\right)(x) \widetilde{v}^{\beta}=0 \text { in } D, \\
\lim _{x \rightarrow z \in \partial D} \frac{\widetilde{u}(x)}{M_{\alpha}^{D} 1(x)}=\varphi(z), \\
\lim _{x \rightarrow z \in \partial D} \frac{\widetilde{v}(x)}{M_{\alpha}^{D} 1(x)}=\psi(z) .
\end{gathered}
$$

According to Theorem 2, we have

$$
\begin{gathered}
\omega=1-\frac{1}{M_{\alpha}^{D} \varphi} V\left(z^{r} \omega^{\sigma}\left(M_{\alpha}^{D} \psi\right)^{r}\left(M_{\alpha}^{D} \varphi\right)^{\sigma} p\right) \\
\theta=1-\frac{1}{M_{\alpha}^{D} \psi} V\left(y^{s} \omega^{\beta}\left(M_{\alpha}^{D} \varphi\right)^{s}\left(M_{\alpha}^{D} \psi\right)^{\beta} q\right) .
\end{gathered}
$$

Moreover, we have $c_{1} \leq \omega \leq 1$ and $c_{2} \leq \theta \leq 1$ and by Lemma 6, $T(\Gamma)$ is equicontinuous on $\bar{D}$. Since $T(\Gamma)$ is also bounded, then we deduce that $T(\Gamma)$ is relatively compact in $C(\bar{D}) \times C(\bar{D})$. This implies in particular that $T(\Gamma) \subset \Gamma$.

Next, we shall prove the continuity of the operator $T$ in $\Gamma$ in the supremum norm. Let $\left(y_{k}, z_{k}\right)_{k}$ be a sequence in $\Gamma$ which converges uniformly to a function $(y, z)$ in $\Gamma$. Put $\left(\omega_{k}, \theta_{k}\right)=$ $T\left(y_{k}, z_{k}\right)$ and $(\omega, \theta)=T(y, z)$. Then, we have

$$
\begin{aligned}
\left|\omega_{k}-\omega\right|= & \mid \frac{1}{M_{\alpha}^{D} \varphi} V\left(z^{r} \omega^{\sigma}\left(M_{\alpha}^{D} \psi\right)^{r}\left(M_{\alpha}^{D} \varphi\right)^{\sigma} p\right) \\
& \quad-\frac{1}{M_{\alpha}^{D} \varphi} V\left(z_{k}^{r} \omega_{k}^{\sigma}\left(M_{\alpha}^{D} \psi\right)^{r}\left(M_{\alpha}^{D} \varphi\right)^{\sigma} p\right) \mid \\
\leq & \frac{1}{\sigma M_{\alpha}^{D} \varphi} V\left(\left|z^{r} \omega^{\sigma}-z_{k}^{r} \omega_{k}^{\sigma}\right|\left(M_{\alpha}^{D} \varphi\right) \tilde{p}\right) .
\end{aligned}
$$

Using the fact that $\left|z^{r} \omega^{\sigma}-z_{k}^{r} \omega_{k}^{\sigma}\right| \leq 2$ and that $\tilde{p} \in K_{\alpha}(D)$, we deduce by Proposition 4 and the dominated convergence theorem, that $\omega_{k} \rightarrow \omega$ as $k \rightarrow \infty$. Similarly, we prove that $\theta_{k} \rightarrow \theta$ as $k \rightarrow \infty$. So, $T\left(y_{k}, z_{k}\right) \rightarrow T(y, z)$ as $k \rightarrow \infty$. Since $T(\Gamma)$ is relatively compact in $C(\bar{D}) \times C(\bar{D})$, we deduce that

$$
\left\|T\left(y_{k}, z_{k}\right)-T(y, z)\right\|_{\infty} \longrightarrow 0 \quad \text { as } k \longrightarrow \infty .
$$


From the Schauder fixed point theorem there exists $(y, z) \in \Gamma$ such that $T(y, z)=(y, z)$ or equivalently

$$
\begin{aligned}
& u=M_{\alpha}^{D} \varphi-V\left(p u^{\sigma} v^{r}\right), \\
& v=M_{\alpha}^{D} \psi-V\left(q u^{s} v^{\beta}\right),
\end{aligned}
$$

where $(u, v)=\left(y M_{\alpha}^{D} \varphi, z M_{\alpha}^{D} \psi\right)$. The pair $(u, v)$ is a required solution of (1) in the sense of distributions. This completes the proof.

\section{Acknowledgment}

The research of Imed Bachar is supported by NPST Program of King Saud University; Project no. 11-MAT1716-02.

\section{References}

[1] A. Pazy, Semigroups of Linear Operators and Applications to Partial Differential Equations, vol. 44 of Applied Mathematical Sciences, Springer, New York, NY, USA, 1983.

[2] K. Yosida, Functional Analysis, vol. 123 of Fundamental Principles of Mathematical Sciences, Springer, Berlin, Germany, 6th edition, 1980.

[3] J. Glover, Z. Pop-Stojanovic, M. Rao, H. Šikić, R. Song, and Z. Vondraček, "Harmonic functions of subordinate killed Brownian motion," Journal of Functional Analysis, vol. 215, no. 2, pp. 399-426, 2004.

[4] J. Glover, M. Rao, H. Šikić, and R. M. Song, "Г-potentials," in Classical and Modern Potential Theory and Applications (Chateau de Bonas, 1993), vol. 430 of NATO Adv. Sci. Inst. Ser. C Math. Phys. Sci., pp. 217-232, Kluwer Acad. Publ., Dordrecht, The Netherlands, 1994.

[5] R. Song, "Sharp bounds on the density, Green function and jumping function of subordinate killed BM," Probability Theory and Related Fields, vol. 128, no. 4, pp. 606-628, 2004.

[6] R. Song and Z. Vondraček, "Potential theory of subordinate killed Brownian motion in a domain," Probability Theory and Related Fields, vol. 125, no. 4, pp. 578-592, 2003.

[7] F.-C. Şt. Cîrstea and V. D. Rădulescu, "Entire solutions blowing up at infinity for semilinear elliptic systems," Journal de Mathématiques Pures et Appliquées, vol. 81, no. 9, pp. 827-846, 2002.

[8] A. Ghanmi, H. Mâagli, S. Turki, and N. Zeddini, "Existence of positive bounded solutions for some nonlinear elliptic systems," Journal of Mathematical Analysis and Applications, vol. 352, no. 1, pp. 440-448, 2009.

[9] M. Ghergu and V. Rădulescu, "On a class of singular GiererMeinhardt systems arising in morphogenesis," Comptes Rendus Mathématique, vol. 344, no. 3, pp. 163-168, 2007.

[10] A. V. Lair and A. W. Wood, "Large solutions of semilinear elliptic equations with nonlinear gradient terms," International Journal of Mathematics and Mathematical Sciences, vol. 22, no. 4, pp. 869-883, 1999.

[11] A. V. Lair and A. W. Wood, "Existence of entire large positive solutions of semilinear elliptic systems," Journal of Differential Equations, vol. 164, no. 2, pp. 380-394, 2000.

[12] C. Mu, S. Huang, Q. Tian, and L. Liu, "Large solutions for an elliptic system of competitive type: existence, uniqueness and asymptotic behavior," Nonlinear Analysis: Theory, Methods \& Applications, vol. 71, no. 10, pp. 4544-4552, 2009.
[13] R. S. Alsaedi, H. M. Mâagli, and N. Zeddini:, "Positive solutions for some competetive elliptic systems," to appear in Mathematica Slovaca.

[14] A. Dhifli, H. Mâagli, and M. Zribi, "On the subordinate killed B.M in bounded domains and existence results for nonlinear fractional Dirichlet problems," Mathematische Annalen, vol. 352, no. 2, pp. 259-291, 2012.

[15] H. Mâagli and M. Zribi, "On a new Kato class and singular solutions of a nonlinear elliptic equation in bounded domains of $\mathbb{R}^{n}$," Positivity, vol. 9, no. 4, pp. 667-686, 2005.

[16] K. L. Chung and Z. X. Zhao, From Brownian Motion to Schrödinger's Equation, vol. 312 of Fundamental Principles of Mathematical Sciences, Springer, Berlin, Germany, 1995.

[17] H. M. Mâagli, "Perturbation Semi-Linéaire des Résolvantes et des Semi-Groupes," Potential Analysis, vol. 3, pp. 61-87, 1994.

[18] J. Bliedtner and W. Hansen, Potential Theory. An Analytic and Probabilistic Approach to Balayage, Universitext, Springer, Berlin, Germany, 1986.

[19] D. V. Widder, The Laplace Transform, vol. 6 of Princeton Mathematical Series, Princeton University Press, Princeton, NJ, USA, 1941. 


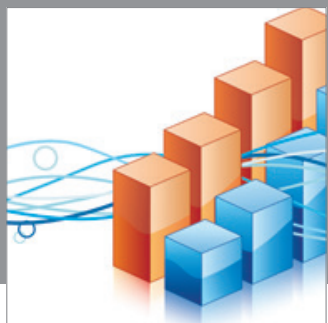

Advances in

Operations Research

mansans

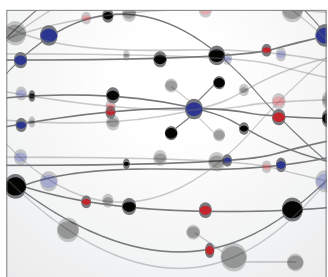

The Scientific World Journal
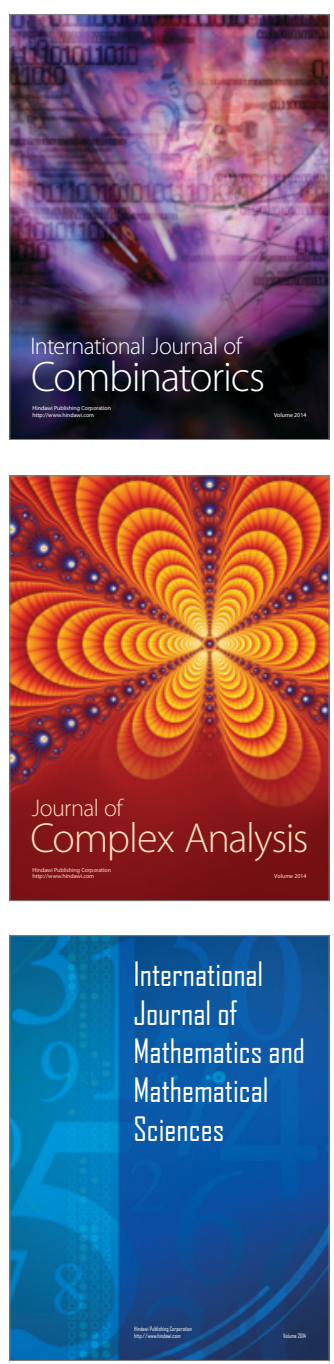
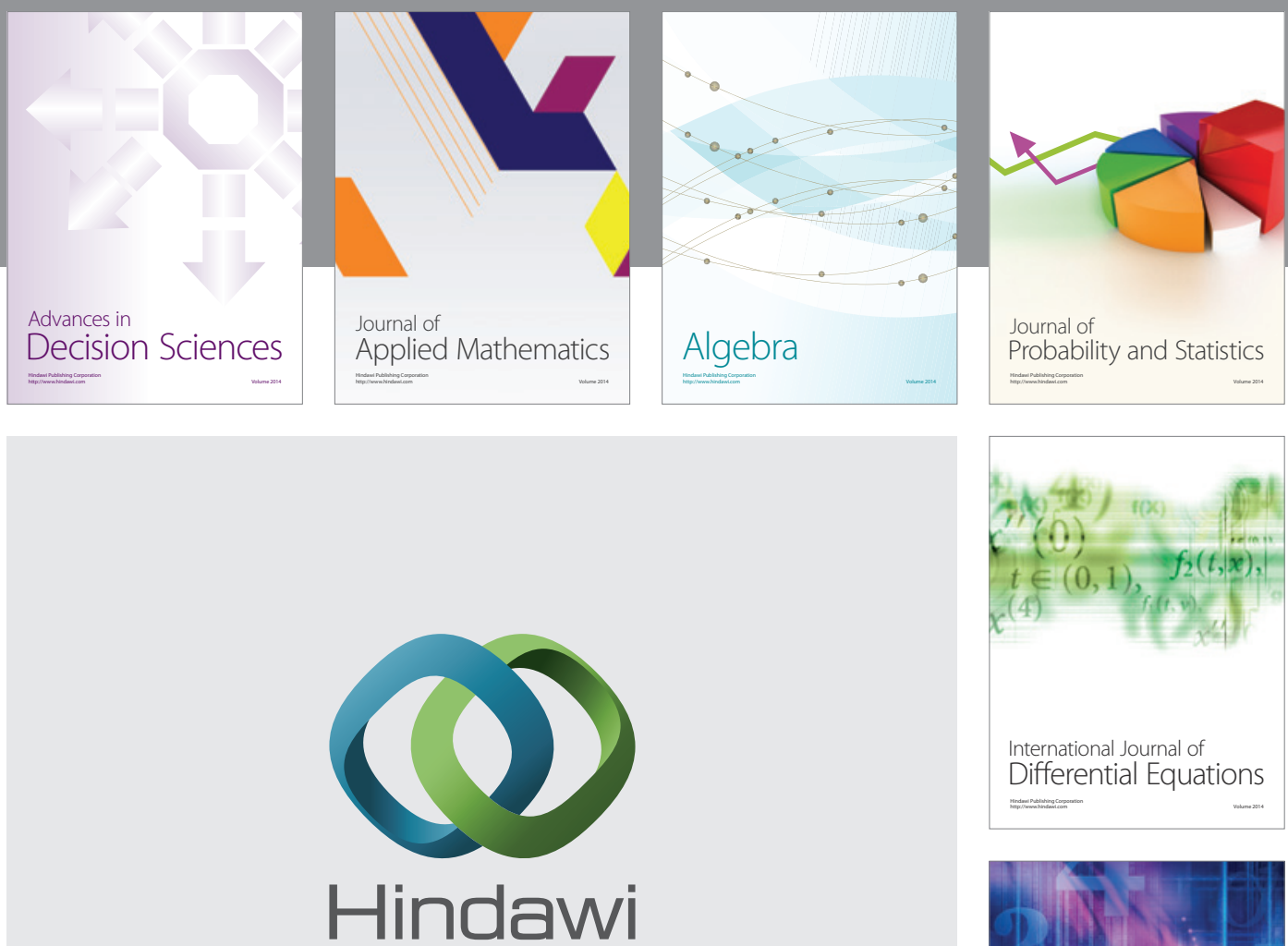

Submit your manuscripts at http://www.hindawi.com
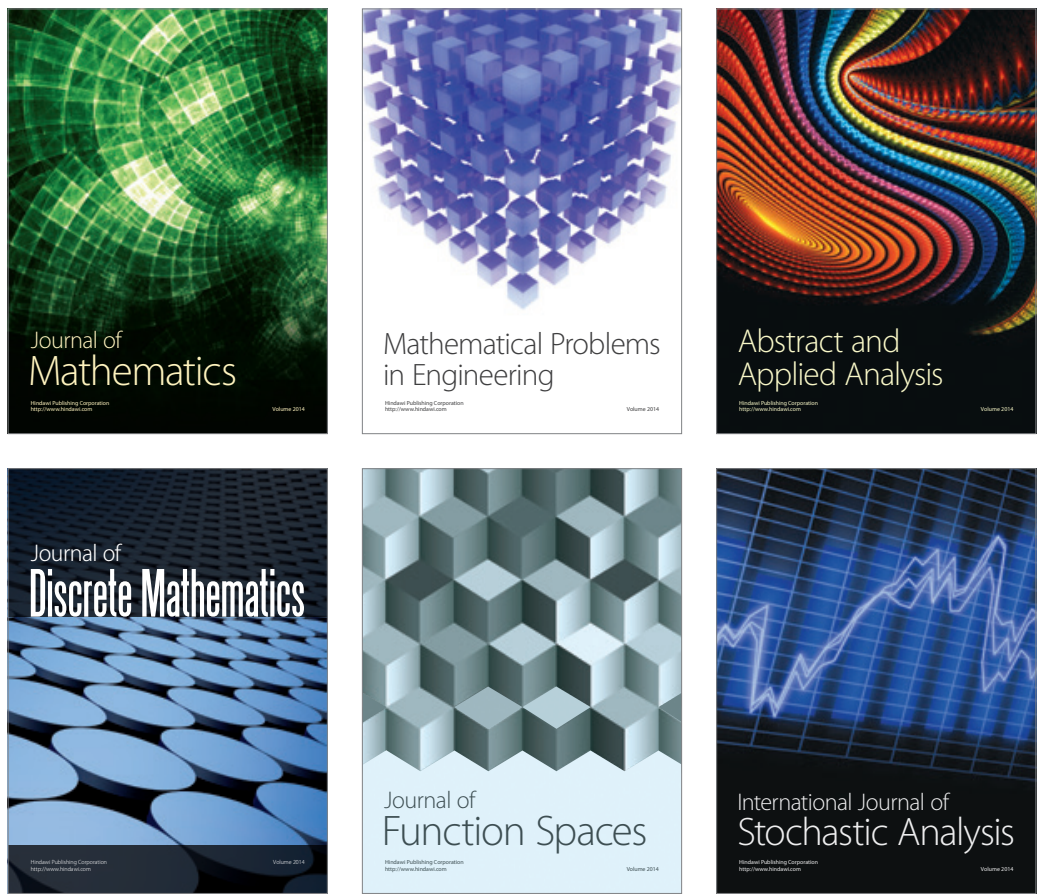

Journal of

Function Spaces

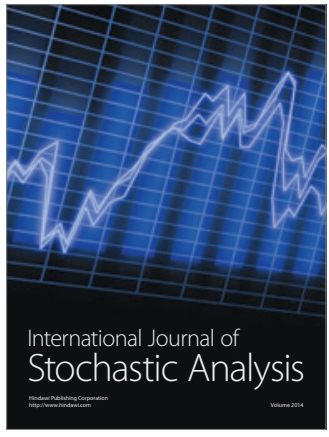

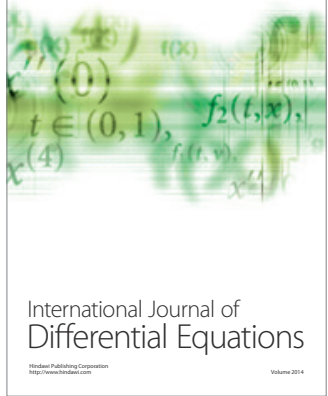
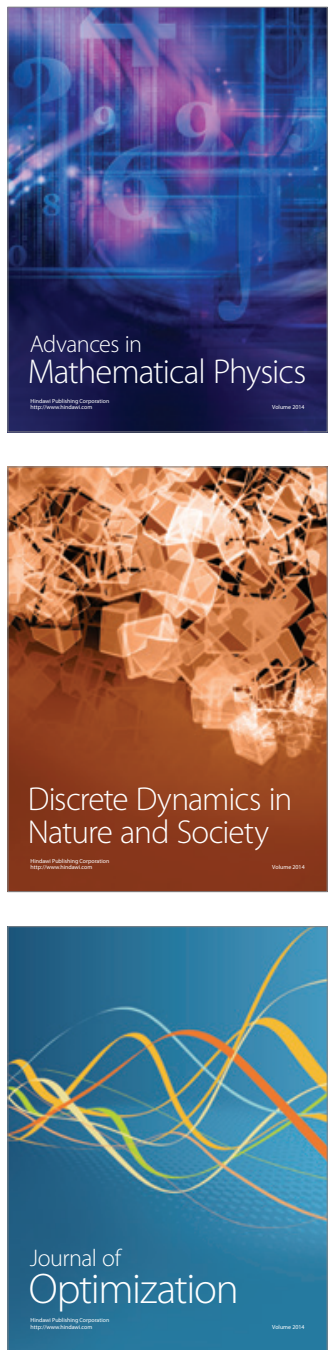